\title{
IDENTIFICATION OF GEOSTATIONARY SATELLITES BY THEIR PHOTOMETRIC AND DYNAMIC FEATURES, SOME PROBLEMS
}

\author{
P.P.Sukhov, K.P.Sukhov \\ Astronomical Observatory, Odessa National University \\ Shevchenko Park, 65014, Odessa, Ukraine \\ psukhov@ukr.net
}

\begin{abstract}
It is possible to identify certain characteristics of geostationary satellites by studying their photometry. This paper reviews some of the technical problems and methodologies concerning photometry of satellites, as well as their identification by photometric characteristics. The existing methods of identifying geostationary satellites using photometry are also summarized. The present Database of photometric and dynamic characteristics of the GSS, additional information, simplifying the input of the inverse problem - all this allows modern methods to identify unknown GSS with a probability up to $80 \%$.
\end{abstract}

Key words: geostationary satellite, GSS, photometrical features, identification, phase angle, effective reflective area, inverse problem.

\section{Introduction}

For geostationary satellites (GSS) identification, often enough orbital characteristics. This six Keplerian elements and the orbital plane position relative to the equatorial plane or Laplacian plane. However, as referred to in [1], in some cases, the identification of a compact groups GSS by its orbital parameters within an orbital slot or by coincidence of the orbital planes of explosion fragments results in erroneous identification. The non-coordinate information, such as photometrical, polarisation and spectral data, can play a crucial part in identification of the type of satellite and its purpose of use in the following cases: 1 . The GSS redeployment, manoeuvres (which are quite frequent) or changeover of the temporarily deactivated satellite back to the active status. 2. Highly elliptical orbits are poorly monitored by ground-based radar and optical aids of space control services. 3. As a result of evolution, due to perturbations by the Moon, Sun, Earth, or light pressure, the GSS orbital plane oscillates relative to the Laplacian plane with a period of 54 years and an amplitude of about $15^{0}$ [1]. Old passive GSS will enter the equatorial plane by 2017. And it will be difficult to distinguish a new GSS from an old one, which was launched 54 years ago, by orbital parameters.

The following methods for space object (SO) identification are known: 1. Identification by orbital parameters.
2. Identification by the image obtained with long focallength optical telescopes. 3. Identification by the space object (SO) image during its passage across the Moon's or Sun's disc. 4. Identification using orbital satellites which monitor and take photographs of the satellite of interest. 5 . Identification by analysing telemetry and radio data transmitted from SO. 6. Identification using a set of reflective characteristics in the radio and optical wavelengths (photometrical, polarization, spectral), which is typical for this class of satellites.

The physical characteristics, obtained from photometrical observations of GSS, used for identification are as follows:

Photometric characteristics. 1. The effective reflecting area $\left(\mathrm{S}_{\gamma \lambda}\right) ; 2$. The spectral reflectance index $\left(\gamma_{\lambda}\right)$. 3. The phase coefficient $\beta$, ( $\Delta m / d e g)) ; 4$. Colour-indices $(C I)$, $(B$ $V, V-R)$. 5. The magnitude $m$ reduced to a standard distance and phase angle (usually to $\psi=0^{0}$ or $\psi=25^{0}$ ).

Opto-geometric characteristics. The GSS linear dimensions. The prevailing shape of an object.

Dynamic characteristics. The period of rotation about the centre of mass or one of the axes. Instantaneous orientation in space, which is defined by the normal to the GSS reflective surface that produces flashes.

Physical characteristics of satellite platforms and payloads are not always known, but they can be determined from photometric observations. Problems with GSS photometry and the use of photometric data for the identification of GSS can be divided into two groups: technical and methodological.

\section{Technical problems}

1. Faint light of GSS: at the phase angle $\psi>30^{\circ}$, the GSS is rather faint as its brightness is around $10^{\mathrm{m}}-15^{\mathrm{m}}$. Therefore, it is advisable to use telescopes with the primary mirror diameter of at least 1 meter. Traditionally, to determine the GSS reflectance profile, it is necessary to conduct observations over a long period of time (from half-year, year) at different GSS positions relative to an observer. Basic observations are often limited due to weather conditions at observation sites.

However, there are some dates when it is possible to use telescopes with primary mirrors of $40-70 \mathrm{~cm}$ in diame- 
ter for the photometry of GSS. Those are dates near the equinoxes, when a GSS enters and exits the Earth's shadow [2-4]. At that time, the GSS apparent brightness increases by $5^{\mathrm{m}}-10^{\mathrm{m}}$ due to the so-called flash before entering and after exiting the Earth's shadow.

At this time, the GSS brightness increases by $5^{\mathrm{m}}-10^{\mathrm{m}}$ due to the flash before entering and after exiting the Earth's shadow.

The flashes are created by quasi-mirror reflection of sunlight from the GSS surface, mainly from the solar arrays. Those flashes can be used to determine the object's payload [5] and orientation in space using the method suggested by V. Yepishev [6]. The number of such nights favourable for observations can be to 80 per year [7].

2. To register possible GSS rapid brightness changes, the obtained photometric measurements must be a minimum temporal resolution, a fraction of a second. In this case it is possible to use CCD run in storage mode for the rough photometry of three-axis stabilised GSSs, which change their brightness slowly. But using such CCD for the photometry of rapidly rotating GSS or space debris will result in smoothing small low-amplitude periodic effects on the light curve. The most relevant light detectors for this purpose are the following: 1) the use of CCD operated in TV mode with mode count pulses; 2) the use of the device immediate action photomultiplier (FEU) operating in the pulse-counting mode. The FEU is a single-channel device, but it has unexcelled advantages in its amplification factor $\left(>10^{6}\right)$ and wide dynamic range $\left(>10^{\mathrm{m}}\right)$.

3. Multicolour photometry of GSS with the rapid changes of brightness; the time resolution is limited by the time the photometer switches the color filter.

\section{Methodological problems}

In the context of mathematical physics, the problem of identification by photometric observations can be solved in two ways.

Direct ways. The problem of calculating the brightness of the object from the known characteristics, such as shape, the laws of reflection, the orientation of the object, etc. In this case, we have a unique solution for any time.

Inverse ways. The determination of the object's shape by its light curve is an ill-posed inverse problem in mathematical physics. It does not correspond to the Hadamard criterion. We know only a part of the input information - brightness depending on the time $\mathrm{F}=m(t)$. The output should be the object's shape as function $F=m(t) \Theta(X, Y, Z)$ with two arguments, i.e. the brightness of the time $m(t)$ and angles of object's orientation $\Theta(X, Y, Z)$. In this case, the obtained results are characterised by the fact that small input changes can yield arbitrary large changes in the obtained results. In general, the solutions will be unstable, ambiguous or physically meaningless. The inverse problem solution depends on a large extent of the availability of a priori (physical) information on the object.

Several methods are used to reduce the reflectance profiles to $\psi=0^{0}$. You can extrapolate the diffuse component of the light curve for $\psi=0^{0}$, using a polynomial approximation. However, is possible to apply algorithm described in [8] where the GSS brightness is expressed by the formula:

$$
m_{\lambda}=m_{\lambda}^{\Theta}-2,5 \lg \left[\frac{S \gamma_{\lambda} F(\psi)}{d^{2}}\right],
$$

where $m_{\lambda}^{\Theta}$-is the magnitude of the Sun in a certain spectral range $\lambda ; S-$ is the GSS surface illuminated by the Sun and visible to an observer; $\gamma_{\lambda}-$ is the spectral reflectance factor; $F(\psi)$ - is the phase function; $\psi-$ is the satellite-centric phase angle; $d-$ is the topocentric distance to the spacecraft; and $S$ $\gamma_{\lambda}-$ is the effective reflecting area (ERA). All variables in the formula, except ERA, can be reliably measured or calculated from observations. $S \gamma_{\lambda}$ is a probabilistic variable. Incorrect determination of this variable leads to errors in calculation of other physical characteristics of GSS.

Experts use different ways of SO identification. They depend on the availability of a priori information about the SO observed. Therefore, the number of criteria for the identification of SO may be different. To identify an SO it is sometimes enough to determine 1-2 dynamic or reflection characteristics.

Let us present a brief overview of the most common methods for GSS identification by photometric data applied by the CIS experts. The methods for GSS identification used by Western experts from other countries are not given in the press.

1. The method for identification of low-orbit and highorbit satellites developed in Uzhgorod, Ukraine, by V.P.Yepishev, I.I.Motrunich, Y.M.Motrunich, I.F.Naybauer et al.- is based on the on the complex approach to problem solving. The photometric data must be used together with positional observation data and colorimetric and polarimetric measurements applied when possible. That allows for determining the orientation of a satellite and individual fragments of its surface not only in space, but also relative to an observer and the GSS orbital plane. The authors developed nine criteria which makes it possible to achieve $80 \%$ probability of unknown satellite identification even when there is no a priori information on the SO [9].

2. The colour-index (CI) of an unknown spacecraft is the key characteristic of the idea developed by A.Murtazov, N.Nosova, V.Kupriyanov et al. (Ryazan, Rissia), as well V.Prokofieva (The Crimean Astrophysical Observatory). This $C I$ is compared with the $C I$ of known types of SOs. The phase coefficient should be applied when there is no similarity between CIs. Further, the comparison with mathematical simulation results for different geometric shapes is drawn and the scattered-field simulation is used $[10,11]$.

3. The methods by A.Didenko, B.Demchenko, L.Usoltseva et al. (Almaty, Kazakhstan). The reference data bank of photometric phase patterns is created for each GSS type. The phase pattern of an unknown GSS is compared with the bank data based on certain criteria. When it is impossible to identify an object, a phase pattern of a new GSS type is created for this object [12]. The identification procedure includes the following parameters: a phase factor $-\beta$, geometric albedo, ERA, spectral reflectance $-\gamma_{\lambda}$, period of light variation. Apply methods of the theory of pattern recognition [13].

4. The method by M.Smirnov, A.Bagrov, V.Vygon et al. (Moscow) are based on the study of the scattered field 
formed by individual elements of the satellite structure. Implementation of this method requires conducting GSS observations over a long period at different phase angles [14]. The mathematical simulation is also applied. A.Bagrov also suggests carrying out GSS spectral observations to estimate the chemical composition of the GSS coating surface [15]. However, it is a sophisticated task to obtain a high-resolution spectrum of a faint GSS; maybe that is why the idea has not obtained a wide circulation.

5. A.Dobrovolsky, A.Korobko et al. (Odessa, Ukraine) have been exploring only the preliminary stage of identification by light curves at which they are divided into two groups: non-periodic and periodic light curves. Each group is broken down into several sub-groups. However, the accordance between the light curve groups and satellite types has not developed [16].

We can see that the solution of the ill-posed problems of GSS identification by photometric data is a long complicated process that requires additional information about the satellite. Such additional information can be the following: the satellite figure (configuration), its dimensions, the launch site and date, the orbit inclination to the equator, solar array power, nose-cone fairing dimensions, etc.

At the beginning of the $21^{\text {st }}$ century, the main satellite systems remained the same as in 1980s. There is not much variety in satellite shapes; the design of their structures has been changed rather slowly. The evolution is driven mainly in towards the improvement of characteristics. As many experts noted, each GSS type has individual design features, which are representative and characterised by the light curve shape - signature. For example, the glare from the scanning optics, the rotation around the axis, the type of stabilization, platform type, the size of the solar panels, and others. These features help to classify the spacecraft according to the light curve. The figures below, without going into the analysis, show the light curves of several geostationary objects with distinctive design and dynamic features characteristic of the respective spacecraft classes. The objects were observed by the authors at the calculated time for the flares maximum amplitude. Figure $1-$ GSS "Sbirs GEO 2". Figure 2 - "DSP 18". Figure 3 - "Mentor 4". Figure 4 - tumble booster "Milstar 5R".

Many experts (see below) for identifying the GSS collect photometric database (DB) containing a set of characteristics with sufficient probability define a specific class of SO.The formation of such a DB is the problem of time and usage of uniform methodology for determining the physical characteristics of the SO. Such characteristics can be the following: ERA, phase coefficient $\beta$, spectral reflectance factors $\left(\gamma_{\lambda}\right), C I$, period of brightness variations, etc.

As proposed in the articles [13, 17], "the effective reflecting area is a rather important criterion, which can be used to identify a GSS type". It is known that the magnitude of the observed GSS mostly depends on two parameters: the ERA $-S \gamma_{\lambda}$ and phase angle $-\psi$. Hence, it may suggest taking ERA as a key characteristic of an SO. The ERA value will allow for the approximate estimation of what type the object belongs to. To simplify calculation of $\mathrm{S} \gamma_{\lambda}$, it makes sense to bring ERA to the phase angle $\psi=$ $25^{\circ}$ or $\psi=30^{\circ}$. At these $\psi$, the GSS brightness varies linearly. The phase coefficient $\beta$ can be another important characteristic.

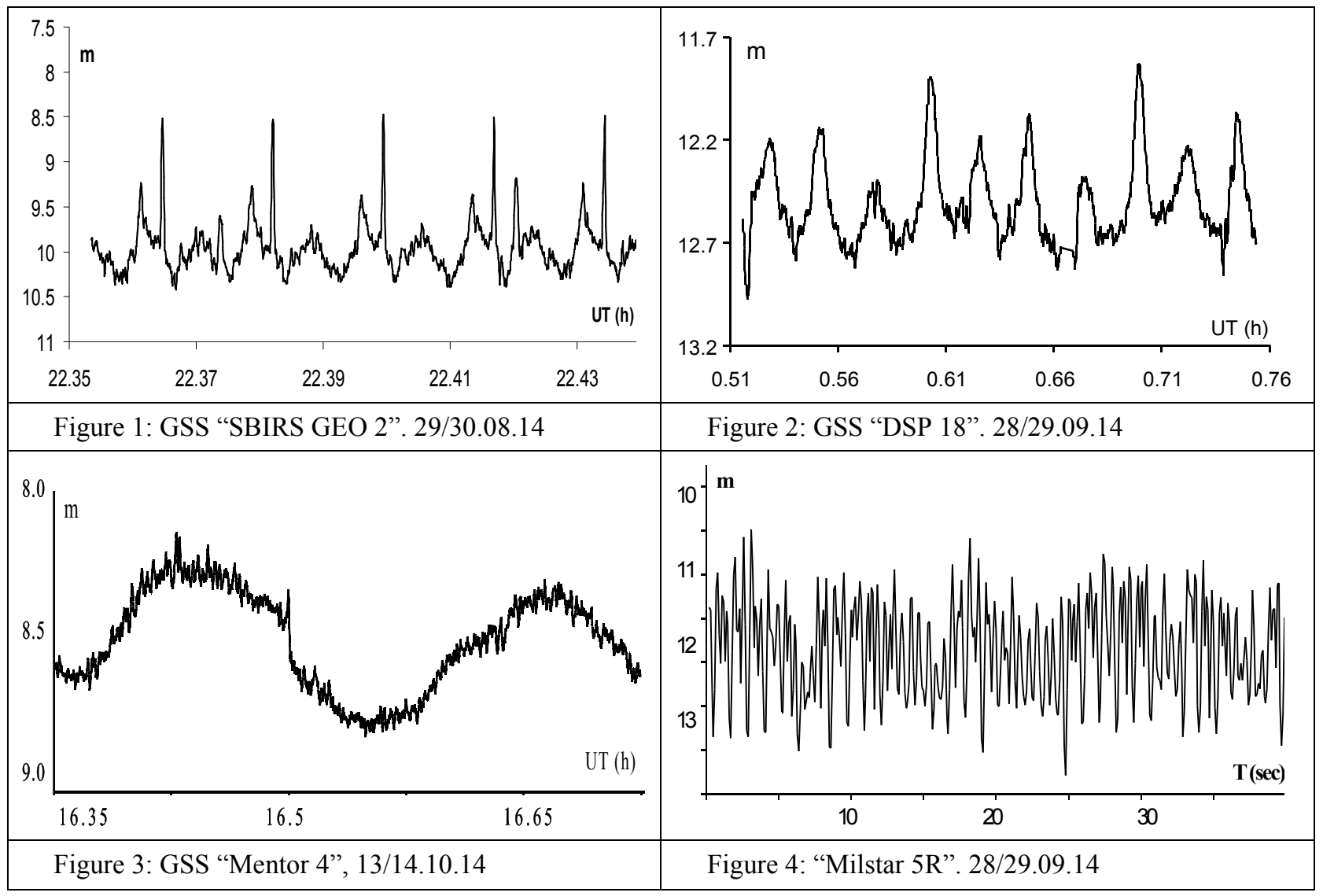


The division of characteristics into primary and secondary is relative and dependent on the availability and sufficiency of additional information on GSS. The further procedure of determination of other GSS physical characteristics depends on the final aim.

The large DB of standardised $(\mathrm{B}, \mathrm{V}, \mathrm{R})$ physical characteristics of many GSS types is created and supported in the Fesenkov Astrophysical Institute (Almaty, Kazakhstan). The similar database of the Astronomical Observatory of I.I.Mechnikov, Odessa National University (Ukraine), contains data on more than 120 GSS and more than 800 light curves, and is updated every year. The "Kosmoten" station (Russia) DB consists of several thousands of light curves for many SO types. On the basis of AF USA - AMOS (Air Force Maui Optical and Supercomputing) intensively carried out are photometric, spectral, and polarization observations of different SO classes $[18,19]$. However, the results of these studies are not known to us.

\section{Conclusion}

The database, additional information and simplification of input data when solving an inverse problem make it possible to identify an unknown satellite with a probability up to $80 \%$ using modern advanced methods [9].

\section{References:}

1. Kiladze R.I., Sochilina A.S. Theory of motion of geostationary satellites, S. Petersburg, 2008, 10.

2. Sukhov P.P, Karpenko G.F., Epishev V.P., Motrunych I.I.: 2009/2010, Odessa Astron. Publ., 22, 55.

3. Didenko A.V., Usol'tseva L.A.: 2002, Kosmicheskiye issledovaniya v Kazakhstane, Almaty: KazGosINTY, 355.

4. Didenko A.V., Usol'tseva L.A.: 2005, Pervyye Fesenkovskiye chteniya «Sovremennaya astrofizika: traditsii $i$ perspektivy», Almaty, 75 (in Russian).

5. Didenko A.V., Usol'tseva L.A.: 2008, Izvestiya NAN RK, Seriya fiziko-matematicheskaya, 4, 96 (in Russian).

6. Yepishev V.P.: 1983, Astrometriya $i$ astrofizika $A N$ SSSR, 89 (in Russian).

7. Karpenko G.F., Murnikov B.A., Sukhov P.P.: 2009/2010, Odessa Astron. Publ., 22, 25.
8. Severnyy S.A., Smirnov M.A., Bagrov A.V.: 1986, Nauchnyye informatsii, 58, M., 103 (in Russian).

9. YepishevV.P., IsaakI.I., Motrunich I.I., Naybauer I.F., Novak E.I., Tsikavyy A.A.: 2005, Sbornik trudov. Okolozemnaya astronomiya, 267 (in Russian).

10. Murtazov A.K. Ekologiya okolozemnogo kosmicheskogo prostranstva, M.: FIZMATLIT, 2004; MAIK «Nauka / Interperiodika», 304 p. (in Russian).

11. Kuryshev V.I., Murtazov A.K.: 1985, Nablyudeniya iskustvennykh nebesnykh tel, 28 (in Russian).

12. Didenko A.V., Usoltzeva. L.A.: 2001, Transaction of the $K A U, 2,83$.

13. Didenko A.V., Usol'tseva L.A.: 2007, Izvestiya NAN $R K$, Seriya fiziko-matematicheskaya, 4, 18 (in Russian).

14. Smirnov M.A. Fotometricheskiye nablyudeniya iskustvennykh nebesnykh tel //Diss. na Soiskaniye uchenoy stepeni doktora fiz.-mat. Nauk, Moskva, 1994, 163 p. (in Russian).

15. Bagrov A.V. Okolozemnaya astronomiya: issledovaniye iskustvennykh i yestestvennykh nebesnet tel $\mathrm{v}$ okolozemnom kosmicheskom prostranstve //Diss. na Soiskaniye uchenoy stepeni doktora fiz.-mat. Nauk, Moskva, 2002, 98-106 (in Russian).

16. Korobko A.A., Dobrovolsky A.V., Medvedev Y.A., et al.: 1999, in Proc. Gamov memorial international conference, Odessa, August 16-22, 122.

17. Yepishev V.P., Karpenko G.F., Sukhov P.P., Klabukova A.V., Volkov S.K.: 2009, Okolozemnaya astronomiya. Sbornik trudov konferentsii, Kazan', 22-26 Aug., M.: GEOS, 2010, 87 (in Russian).

18. Nishimoto D., Kissel K.E., Africano J.L., Lambert J.V., Kervin P.W.: 1993, in: Proc. of the 1993 Space Surveillance Workshop. Project Report STK-206, Vol. $1 / /$ Ed. R.W.Miller \& R.Sridharan, Lincoln Laboratory Massachusetts Institute of Technology, F19628-90-C0002, p. 1-10.

19. Africano J., Kervin P., Sydney P., Hamada K., Soo Hoo V., Nishimoto D.: 2001, in: Proc. of the Third European Conference on Space Debris, 19-21 March 2001, Darmstadt, Germany //Ed. Huguette Sawaya-Lacoste. ESA SP473, Vol.1, Noordwijk, Netherlands: ESA Publications Division, p. 107-112. 